\section{Hohes Alter kein Grund, Prostatakrebs nicht zu therapieren}

\begin{abstract}
Entscheidungen zur Therapie des Prostatakarzinoms werden oft auf Basis des Alters der Patienten getroffen. Zu Unrecht, wie eine aktuelle Studie belegt.
\end{abstract}

U S-amerikanische Wissenschaftler wollten herausfinden, wie häufig ältere Prostatakarzinom-Patienten im Vergleich zu jüngeren eine interventionelle Therapie erhalten, und zwar abhängig vom Fortschritt der Krankheit. Sie bezogen in ihre Studie Daten von 11.790 Pati-

enten aus der CaPSURE (Cancer of the Prostate Strategic Urologic Research Endeavour)-Datenbank ein, um den $\mathrm{Zu}$ sammenhang von Alter, Krankheitsrisiko und prostatakrebsspezifischem Gesamtüberleben zu klären. Zu den Behandlungen zählten auf der einen Seite Prosta-

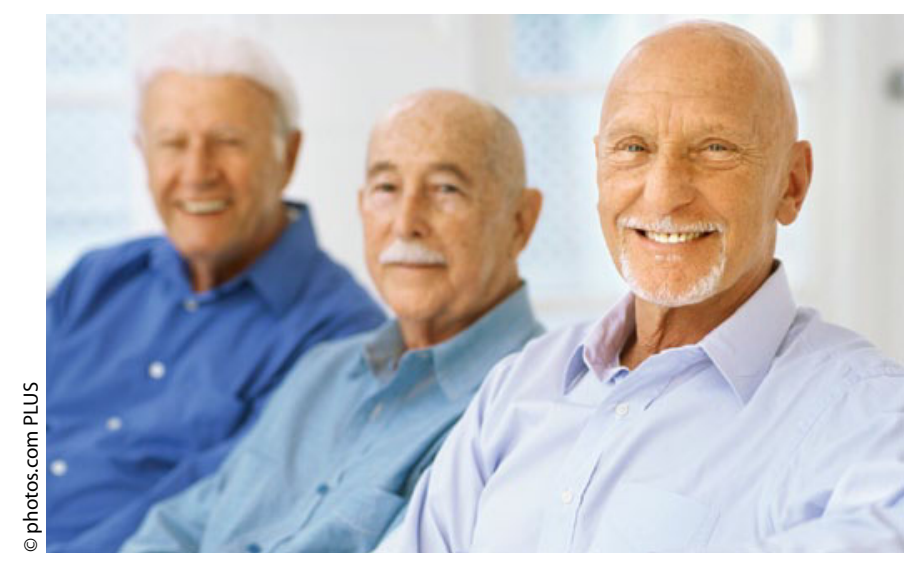

Nicht zu alt für eine Therapie: Patienten über 70 haben keine höhere prostatakrebsspezifische Mortalität als $\mathrm{Pa}$ tienten unter 55 . tektomie, Kryotherapie, Strahlentherapie und Brachytherapie, auf der anderen Seite Androgendeprivationstherapie und Active Surveillance.

Nach Bereinigung um Behandlungstyp und Ausgangsrisiko haben Patienten über 70 keine höhere prostatakrebsspezifische Mortalität als Patienten unter 55. Darüber hinaus war bei älteren Patienten mit Hoch-Risiko-Prostatakarzinom die Mortalität um 46\% reduziert, wenn sie interventionell und nicht konservativ behandelt wurden (OR 0,54, 95\% CI 0,41-0,72).

Fazit: Die Autoren schließen aus diesen Ergebnissen, dass eine Untertherapie von Patienten über 75 Jahren ein genauso wichtiges Problem ist wie eine Übertherapie von Niedrig-Risiko-Patienten. Sie empfehlen, das Alter bei der Therapieentscheidung zu vernachlässigen und ältere Männer einer Prostatektomie oder einer Strahlentherapie zu unterziehen, wenn sie noch eine Lebenserwartung von über zehn Jahren haben.

Bechis $S$ et al. The impact of age at diagnosis on prostate cancer treatment and survival. J Clin Oncol 2011; 29: 235-41

\section{Blasenkarzinom: \\ Gensignatur sagt Metastasie-Risiko voraus}

\section{Die Prognose einer jeden Krebserkrankung wird maßgeblich davon beeinflusst, ob der Tumor metastasiert oder nicht. Anhand einer bestimmten Gensignatur lässt sich nun das Risiko eines jeden Blasenkarzinom-Patienten individuell bestimmen. So können Hochrisiko-Patienten identifiziert und eventuell mit einer neoadjuvanten Therapie ihre Überlebenschancen verbessert werden.}

$\mathrm{D}$ ie Bestimmung der Expression von zwanzig Genen kann vorhersagen, ob bei Blasenkarzinomen Metastasen in den Lymphknoten auftreten. Das wiesen Erlanger Wissenschaftler in einer Kooperation mit Kollegen von der Universität Colorado sowie der Universität Virginia in den USA und der Laval-Universität in Quebec/Kanada nach.

Der US-amerikanischen Arbeitsgruppe gelang es zunächst, eine Gensignatur zu identifizieren, welche die Metastasierung des Blasenkarzinoms vorhersagen konnte. Die Wissenschaftler haben dabei die
Methodik so optimiert, dass die Expression dieser Gene auch am Formalin-fixierten und in Paraffin eingebetteten Routinematerial aus der Pathologie möglich wurde. Daraufhin untersuchten die Erlanger Kollegen, ob diese Gensignatur die Lymphknotenmetastasen auch in einer großen prospektiven Studienkohorte identifizieren konnte, die vor 15 Jahren durch die Arbeitsgemeinschaft Urologische Onkologie der Deutschen Krebsgesellschaft untersucht wurde.

Die Ergebnisse sind vielversprechend: Es besteht eine signifikante Korrelation zwischen der Gensignatur und späterer Lymphknotenmetastasen sowie dem Überleben der Patienten. Das eröffnet möglicherweise eine große therapeutische Option: Denn Patienten, bei denen anhand der Genexpression ein hohes Risiko vorliegt, könnte dann bereits vor ihrer Operation eine zusätzliche Chemotherapie angeboten werden, um mögliche, aber noch nicht nachweisbare Mikrometastasen zu bekämpfen.

Fazit: Ersten klinischen Studienergebnissen zufolge kann eine neoadjuvante Therapie zumindest bei manchen Harnblasenkarziom-Patienten von großem Nutzen sein. Prospektive Studien müssen klären, ob dieses Vorgehen das Überleben der Patienten tatsächlich verbessert. $\mathbf{d k}$

Smith S. C. et al. A 20-gene model for molecular nodal staging of bladder cancer: development and prospective assessment. Lancet Oncol 2011; 12: 137-43 